\title{
Implantação do núcleo de segurança do paciente: ações de capacitação e desenvolvimento institucional
}

\author{
Implementation of the patient safety core: institutional development and capacity \\ building actions
}
Implantación del núcleo de seguridade del paciente: acciones de capacitación y desarrollo institucional

Francisco Alves Lima Júnior ${ }^{1,2,5^{*}}$, Mauro de Souza Pantoja1 ${ }^{1}$ Karla Vanessa Morais Lima ${ }^{2,3}$, Raquel Machado Borges ${ }^{2,3}$, Alexsander Silva de Oliveira ${ }^{4}$, Arlane Silva Carvalho Chaves ${ }^{4}$, Raidanes Barros Barroso $^{4}$, Valteir Conceição da Silva ${ }^{5}$.

\section{RESUMO}

Objetivos: Descrever a experiência de implantação do Núcleo de Segurança do Paciente em uma Unidade Hospitalar no Estado do Maranhão. Métodos: Estudo descritivo, do tipo relato de experiência, a partir da implantação do Núcleo de Segurança do Paciente em uma unidade hospitalar, situada na Cidade de Imperatriz-MA. Relata-se de fevereiro de 2017 a maio de 2018. Para escolha temporal considera-se a finalização da primeira etapa de capacitação. Resultados: A Instalação e posse da Comissão do Núcleo de Segurança do Paciente foi promulgada pela portaria 03/2017, sendo a criação dos documentos-base e protocolos realizada pelos participantes da comissão (Representante dos setores da unidade), para não se tornar uma imposição, garantindo que todos tenham participação ativa na construção destes. No tangente ao uso de tecnologias para melhoria institucional, o hospital utiliza software de saúde para gerenciamento das atividades administrativas e assistenciais. A disseminação de cultura de segurança foi iniciada pelo "evento de lançamento do NSP", dando continuidade com DDS nos setores de atuação, abarcando toda equipe multiprofissional. Conclusão: Depreende-se, por intermédio deste relato, que o processo descrito e seus protocolos é algo contínuo, variando conforme a realidade de cada instituição.

Descritores: Segurança do Paciente, Gestão de Risco, Capacitação em Serviço.

\begin{abstract}
Objectives: To describe the experience of implementation of the Patient Safety Nucleus in a Hospital Unit in the state of Maranhão. Methods: A descriptive study, an experience report type, from the implementation of the Patient Safety Nucleus in a hospital unit, located in the city of Imperatriz-MA. It is reported from February 2017 to May 2018. For temporal choice, the completion of the first qualification stage is considered. Results: Installation and possession of the Committee of the Patient Safety Nucleus was promulgated by ordinance 03/2017, with the creation of the base documents and protocols carried out by the committee participants (Representatives of the unit sectors), so as not to become an imposition, ensuring that all have an active participation in the construction of these. Regarding the use of technologies for institutional improvement, the

\footnotetext{
1 Universidade do Estado do Pará, Programa de Pós-graduação em Cirurgia e Pesquisa Experimental UEPA/CIPE, Belém-PA;

${ }^{2}$ Faculdade de Ciências Médicas e Jurídicas, Faculdade do Bico do Papagaio FACMED/FABIC, Augustinópolis-TO;

${ }^{3}$ Faculdade de Imperatriz - FACIMPM WYDEN, Imperatriz-MA;

4 Universidade Federal do Maranhão - UFMA, Campus Imperatriz-MA;

${ }^{5}$ Hospital Macrorregional Dra. Ruth Noleto, Imperatriz-MA. *E-mail: francisco.enfdotrabalho@gmail.com
} 
hospital uses health software to manage administrative and care activities. The dissemination of safety culture was initiated by the \&quot;PSN launch event\&quot;, continuing with DDS in the sectors involved, encompassing all multiprofessional teams. Conclusion: It is clear through this report that the process described, and its protocols is something continuous, varying according to the reality of each institution.

Descriptors: Patient Safety, Risk Management, Inservice Training.

\section{RESUMEN}

Objetivos: Describir la experiencia de implantación del núcleo de seguridad del paciente en una unidad hospitalaria en el estado de Maranhao. Métodos: Estudios descriptivos, del tipo relato de experiencia, a partír de la implantación del núcleo de seguridad Del paciente en una unidad hospitalaria, situada en la ciudad de Imperatriz-MA. Se relata de febrero de 2017 a mayo de 2018. Para la elección temporal se considera la finalización de la primera etapa de capacitación. Resultados: La instalación y la posesión de la Comisión del núcleo de seguridad del paciente, fue promulgada por la ordenanza 03/2017, siendo la creación de la base de documentos y protocolos realizados por los participantes de la Comisión (Representantes de los sectores de la Unidad) para no convertirse en una imposición, garantizando que todos tengan participación activa en la construcción de estos. En la tangente, al uso de tecnología para la mejora institucional, el hospital utiliza software de salud para la gestión de las actividades administrativas y asistenciales. Se inició la diseminación de cultivo de seguridad por el evento de lanzamiento en el NSP, dando continuidad con DDS en los sectores de actuación, abarcando todo el equipo multiprofesional. Conclusión: Se desprende por intermedio de este relato, que el proceso descrito y sus protocolos es algo continuo, que varían según la realidad de cada institución.

Descriptores: Seguridad del Paciente, Gestión de Riesgos, Capacitación en servicio.

\section{INTRODUÇÃO}

A Política Nacional de Segurança do Paciente foi instituída pelo Ministério da Saúde por meio da Portaria $\mathrm{n}^{\circ} 529 \mathrm{em} 1^{\circ}$ de abril de 2013, objetivando melhorar a qualidade dos serviços de saúde com contribuições maciças na segurança do paciente e tornando-a obrigatória nas instituições de saúde no território Nacional (ANVISA, 2017).

Tal programa tem como base seis protocolos básicos que devem ser instituídos e gerenciados em cada Instituição, são eles: identificação do paciente, comunicação eficaz em serviços saúde, segurança na prescrição, uso e administração de medicamentos, higienização das mãos, prevenção de quedas e lesão por pressão e assegurar cirurgia em local de intervenção, procedimento e pacientes corretos (BRASIL, 2013a).

Brasil (2013a), ainda contempla que as ações de segurança do paciente a serem instituídas em serviços de saúde são gerenciadas pela RDC n 36, publicada em 25 de julho de 2013.

No ano de 2016 encontravam-se registrados 2.286 Núcleos de Segurança do Paciente junto a Agência Nacional de Vigilância Sanitária - ANVISA, havendo 53.997 notificações incidentes/eventos adversos em saúde (BRASIL, 2013b).

Estratégias simples como a instituição do Núcleo de Segurança do Paciente (NSP), os protocolos básicos e educação permanente ajudam na prevenção e redução de riscos advindos da assistência, pois funcionam como barreiras de proteção. Além de tudo, ajudam na qualidade da assistência e diminuição de gastos hospitalares (OLIVEIRA, 2014).

Com o intuído de promover a implementação de ações centradas para a segurança do paciente, o Núcleo de Segurança do Paciente (NSP) é um dos componentes mais importantes para a qualidade da assistência nos serviços de saúde, uma vez que busca a melhoria contínua dos processos de cuidado e do uso de 
tecnologias da saúde, a disseminação sistemática da cultura de segurança, articulação e a integração dos processos de gestão de risco e garantia das boas práticas de funcionamento do serviço de saúde (BRASIL, 2016).

Em meio a fragilidade do sistema e saúde do Brasil, somada a falta de segurança do paciente vista em outrora, o NSP veio para prevenir, monitorar e reduzir a ocorrência de eventos adversos que ocorrem na assistência, sendo indispensável a instalação do NSP nas instituições de saúde, pois este por meios de protocolos básicos de segurança do paciente, desenvolvimentos de ações que buscam identificar e prevenir riscos está possibilitando avanços significantes na assistência segura e livre de danos ao paciente (AZEVEDO et al., 2016).

A não instalação do NSP constitui-se não semente uma infração sanitária, como também acarreta grandes prejuízos na assistência ao paciente, com consequências negativas para instituição, tornado os pacientes mais suscetíveis a danos e eventos adversos em decorrência da falta do NSP. Instituições que vivem está triste realidade apresentam erros na assistência, acarretando em uma assistência falha aos pacientes, elevando custos da hospitalização, aumento de eventos adversos, seja por falta de protocolos e/ou educação aos profissionais, em vista deste olhar menos amplo para com a segurança do paciente (AZEVEDO et al., 2016; SIMAN; BRITO, 2016).

A partir de tais fatos, torna-se evidente a necessidade de Implantação e desenvolvimento das ações do Núcleo de Segurança do Paciente nas Instituições de Saúde, tendo em vista que ainda há desconhecimento dos protocolos básicos por parte dos profissionais da área.

Sendo assim, o objetivo do presente artigo é descrever a experiência de implantação do NSP em uma Unidade Hospitalar no Estado do Maranhão, citando suas principais ações, tecnologias utilizadas, obstáculos e métodos de capacitação.

\section{METODOLOGIA}

Estudo descritivo, do tipo relato de experiência, a partir da implantação do Núcleo de Segurança do Paciente em um Hospital situado na Cidade de Imperatriz-MA, na Região Nordeste do Brasil.

O período relatado é referente a fevereiro de 2017 a maio de 2018. A escolha do tempo para fechamento do relato se dá pelo fato de ser considerada a finalização da primeira etapa de capacitação e discussão multiprofissional para atuação e desenvolvimento dos serviços de saúde.

O Hospital em questão é uma instituição de referência cirúrgica para quarenta e três Municípios no Sul do Estado do Maranhão. Possuindo um total de cento e quatro leitos de clínica médica e cirúrgica; Unidade de Terapia Intensiva, dez leitos e Centro Cirúrgico com quatro salas cirúrgicas e três leitos de Recuperação PósAnestésica (RPA), o mesmo foi inaugurado em outubro de 2016.

As especialidades médicas que contam nos serviços são: Clínica médica, cirurgia geral, cirurgia ginecológica, ortopédica, otorrinolaringológica, neurológica, cardiológica, vascular e oftalmológica. É válido destacar que boa parte dos procedimentos cirúrgicos contam com apoio da cirurgia guiada por vídeo.

O hospital dispõe de ambulatório para consulta das especialidades supracitadas e atendimento para avaliação de risco cirúrgico com anestesista e cardiologista.

\section{RESULTADOS E DISCUSSÃO}

\section{Construção de Documentos-base e Tecnologias Utilizadas}

A Instalação e posse da Comissão do Núcleo de Segurança do Paciente se deu pela portaria 03/2017, publicada no dia 17 de fevereiro de 2017, tendo como Presidente o Coordenador da Equipe Multiprofissional e representante dos setores e serviços do hospital como membros, entre eles: supervisão de fisioterapia (Vice-presidente), diretor técnico, diretor administrativo, médica infectologista, enfermeira responsável pelo 
Serviço de Controle de Infecção Hospitalar (SCIH), coordenação do núcleo de qualidade, enfermagem, e supervisões: ambulatório. alas de internação, Unidade de Terapia Intensiva (UTI), centro cirúrgico e Central de Material e Esterilização (CME), farmácia central e satélite, Serviço de Nutrição e Dietética (SND), Serviço de Engenharia de Segurança e Medicina do Trabalho (SESMT), núcleo interno de regulação e recepção.

Na instituição da Comissão é de suma importância a inclusão de representante de cada setor ou categoria do hospital (Equipe Multiprofissional), pois é desta forma que a disseminação de cultura de segurança começará na unidade, tornando o serviço mais acessível aos profissionais e tendo principalmente uma visão crítica relacionada às problemáticas e dificuldades destes.

A primeira reunião teve com objetivo principal apresentar o NSP aos membros da equipe e realizar a divisão de atividades pertinentes à criação de documentos para alicerce do núcleo.

Na reunião supramencionada já houve apresentação do Regimento Interno e Plano de Ação de Segurança do Paciente, construído pelo presidente. A partir da discussão gerada e observação dos objetivos da comissão, houve a divisão das atividades administrativas e assistenciais.

Para tal, decidiu-se que os participantes da comissão e especialistas de cada área construiriam os protocolos básicos sugeridos pelo Ministério da Saúde, assim, o protocolo de risco de lesão por pressão foi entregue para supervisão de fisioterapia; segurança na prescrição, uso e administração de medicamentos para supervisão da farmácia, cirurgia segura para supervisão do centro cirúrgico (o mesmo já se encontrava em pleno funcionamento no setor); higienização das mãos para responsável do SCIH (já implantado); além destes, foram desenvolvidos risco de hemotransfusão pela hematologista e risco de broncoaspiração pela supervisão do SND.

Os protocolos restantes foram desenvolvidos pela coordenação da Comissão, tais como: identificação do paciente, risco de quedas e notificação de eventos adversos. Todos os protocolos passaram por revisão do Núcleo de Segurança do Paciente, coordenação do Núcleo de Qualidade e Direção Técnica, como também por avaliação da médica infectologista (protocolo de higienização das mãos).

É interessante destacar que a criação de documentos de setor deve propiciar a participação de todos os envolvidos, para não se tornar uma imposição, garantindo que todos tenham ciência dos documentos relativos à comissão. Quando há participação ativa, as atividades começam a ter um maior valor agregado, e consequentemente, proporcionando maior aceitação na implantação de tais protocolos.

Para se construir uma assistência mais segura é necessário a participação de toda equipe multiprofissional, gestão e do próprio usuário. A criação da cultura de segurança em instituições de saúde é um processo lento, pois há uma fragilidade desta concepção desde a academia, sendo necessário uma construção de gestão de risco eficaz e envolvendo a todos (AZEVEDO et al. 2016)

No que se refere ao uso de tecnologias para melhoria institucional, o presente hospital utiliza software de saúde (sistema de processamento de transação) para gerenciamento de todas as atividades administrativas e assistenciais.

Tal sistema dar margem ao NSP operacionalizá-lo, assim, serão descritas as atividades pertinentes à Segurança do Paciente: O cadastro e identificação do paciente é realizada com o uso de pulseira com código de barras e placa de gerenciamento de risco com no mínimo três identificadores: nome completo, nome da mãe e data de nascimento.

Quando se trata de avaliação de risco o mesmo tem a Escala de Braden (risco de lesão por pressão) e Escala de Morse (risco de quedas), obtendo o resultado junto ao score no final do seu preenchimento, podendo o profissional migrar para outra tela do sistema e realizar o Diagnóstico de Enfermagem em risco de lesão por pressão ou risco de quedas, já prescrevendo os cuidados necessários.

No que diz respeito à segurança de medicamentos, tal sistema é dotado de prescrição eletrônica e dispensação em dose única e por código de barras, fazendo assim com que evite eventos adversos de troca de medicamentos, dosagem e distribuição errada de modo a comprometer a segurança dos pacientes. 
Para o Protocolo de Cirurgia Segura foi adotado o Checklist disponibilizado pelo MS e adaptado para realidade local, sendo desenvolvido pela equipe no Centro Cirúrgico, de fácil entendimento e preenchimento, porém, o mesmo foi desenvolvido em impresso próprio, não havendo-o no software.

GAVA et al. (2016) relata que a inserção de tecnologias permite uma maior eficiência no cuidado ao paciente, melhora as condições de trabalho, organiza coletas de dados e principalmente amplia a capacidade de gestão das políticas de saúde.

Assim, obtinha-se relatórios através do sistema no que diz respeito à classificação da escala de Braden, escala de Morse e identificação correta do paciente, dando margem para detecção de possível inconformidade e correção das mesmas.

A tecnologia é uma ferramenta indispensável e fundamental para melhoria da segurança do paciente em instituições de saúde, pois ela garante menor risco de eventos adversos, melhorando a qualidade da assistência e dando maior suporte aos profissionais deste setor, podendo diminuir o tempo de internação e assim impactando diretamente nos gastos hospitalares (CASSIANI et al. 2009)

As visitas/auditorias do NSP acontecem pela manhã, onde o responsável participa da visita multiprofissional na UTI e visita leito-aleito nas unidades de internação, tendo como objetivo: verificação da pulseira de identificação e placa de gerenciamento de risco (nome legível, erros de sobrenome e três dígitos identificadores, placa de risco de alergia e jejum), orientação do paciente e familiares enquanto ao conhecimento das medicações administradas, possíveis incidentes e/ou eventos adversos ocorridos durante a internação e avaliação dos prontuários físicos.

Tal atividade permite ao responsável técnico do serviço uma visão mais ampla da implantação dos protocolos, verificando se está ou não em conformidade com a cultura de segurança da instituição, reconhecimentos de possíveis incidentes, podendo agir para prevenção de riscos e identificação de eventos adversos não notificados.

Em Novembro de 2017 a presente Instituição foi selecionada para a "Colaborativa Melhorando a Segurança do Paciente em Larga Escala no Brasil” juntamente a 120 instituições hospitalares em todo território nacional, tal projeto é desenvolvido pelo Ministério da Saúde junto aos hospitais de Excelência no Brasil, sendo eles: Sírio-Libanês, Albert Einstein, Hospital do Coração, Moinho dos Ventos, Oswaldo Cruz e Beneficência Portuguesa e visa diminuir as Infecções Relacionadas à Assistência à Saúde (IRAS).

Em maio de 2018 também foi selecionado para participação do "Projeto de Implantação Nacional de Estratégia Multimodal de Melhoria de Higiene das Mãos em Serviços de Saúde para a Segurança do Paciente", projeto este da Organização Mundial da Saúde (OMS) em apoio das Vigilâncias Sanitárias Nacional e Estaduais.

\section{Implantação dos Protocolos de Segurança do Paciente - Processo Ensino-Aprendizagem}

A primeira atividade de educação continuada promovida pelo NSP foi de "Lançamento do Núcleo de Segurança do Paciente", desenvolvida de 20 a 24 de março de 2017, cuja abordagem foi direcionada para a política e conceitos básicos de segurança do paciente, assim, despertando o olhar crítico para temática em questão.

Nesta primeira abordagem foram convidados todos os funcionários do hospital e empresas terceirizadas a participarem, assim, alcançando o maior contingente possível de servidores. É válido expor que durante este primeiro evento do NSP, o hospital tinha um menor contingente de pessoas, duas alas de Internação que ainda não estavam funcionando, diminuindo assim, cinquenta e dois leitos.

Para o desenvolvimento de tal atividade foram selecionados dois horários por dia, sendo um em cada turno (matutino e vespertino) para o desenvolvimento da atividade educativa, ocorrendo dentro de uma semana corrida, portanto, abrangendo todas as equipes plantonistas, no início foi desenvolvido uma dinâmica de acolhida e interação multiprofissional, mostrando a necessidade de interação entre categorias (Tabela 1). 
Tabela 1 - Participação dos setores na Disseminação de Segurança do Paciente.

\begin{tabular}{lccc}
\hline \multicolumn{1}{c}{ Setores } & Público Esperado & Participantes & \% de participação \\
\hline Internação & 57 & 38 & $67 \%$ \\
\hline UTI & 35 & 14 & $40 \%$ \\
\hline Centro Cirúrgico e CME & 61 & 28 & $46 \%$ \\
\hline Farmácia & 32 & 14 & $44 \%$ \\
\hline Multiprofissional & 34 & 18 & $53 \%$ \\
\hline Serviços Gerais & 44 & 26 & $59 \%$ \\
\hline Administrativos & 28 & 26 & $93 \%$ \\
\hline TOTAL & $\mathbf{2 9 1}$ & $\mathbf{1 6 4}$ & $\mathbf{5 6 \%}$ \\
\hline
\end{tabular}

Fonte: Dados da pesquisa, 2017.

Com a realização deste evento inicial obteve-se uma avaliação do método utilizado para disseminação do conhecimento, assim, o evento mostrou-se produtivo em gerar questionamentos, discussões acerca do que se tratava e quais os passos para obter-se a tão esperada Segurança do Paciente.

Porém, pelo fato da discussão/palestra ser realizada por uma só pessoa, a mesma se tornou cansativa ao longo da semana, causando estresse, fadiga vocal e rouquidão, abrindo o leque para discussões posteriores, na realização de atividades educativas.

Logo após o desenvolvimento deste evento, iniciou-se a implantação dos protocolos básicos da segurança do paciente.

Como metodologia, foi acordado em reunião, a participação dos responsáveis pela criação dos protocolos para prática de ensino, tendo em vista que o mesmo não aconteceria mais em um local reservado e sim nos postos de trabalho, adotando o método utilizado pela Saúde Ocupacional, o Diálogo Diário de Segurança (DDS).

Este método permite ao responsável pela atividade tratar o assunto durante a jornada de trabalho, retirando-o por alguns minutos para discussão da nova sistemática de trabalho, assim, tratando o protocolo de forma prática e adequando-o à realidade daquele setor.

Foi traçado um cronograma para que não chocassem os horários de capacitações dos protocolos e não saturassem os setores de informação, assim, viabilizando a absorção do conteúdo e discussão posterior no retorno ao setor.

Observou-se com este novo método, um maior alcance dos setores, diminuição do tempo proposto para discussão e apresentação de protocolos de modo enxuto e eficaz. Os resultados alcançados por essas capacitações estão explícitos no Gráfico 1, onde foi examinada a quantidade de participantes em cada protocolo, levando-se em consideração o total de funcionários na Instituição.

Já os protocolos com menor participação, tais como: risco de broncoaspiração (51), risco de quedas (112) e segurança na prescrição (169), foram apresentados aos funcionários como atuação direta aos pacientes, principalmente voltado à equipe de enfermagem e multiprofissional.

Pode-se pontuar algumas questões que comprometem as práticas de educação continuada. Em primeira análise, nota-se a não participação de todos os funcionários no processo de ensino-aprendizagem, tendo em vista a movimentação do setor, quantidade de paciente, faltas, trocas de plantão, entre outros motivos e em segunda análise, percebe-se a cultura dos profissionais na aceitação deste processo de construção de conhecimento. 
Gráfico 1 - Apresentação do Alcance da Capacitação dos Protocolos do Núcleo de Segurança do Paciente no Hospital Macrorregional Dra Ruth Noleto, no ano de 2017-2018.

\section{ALCANCE DA CAPACITAÇÃO DE PROTOCOLOS DO NSP}

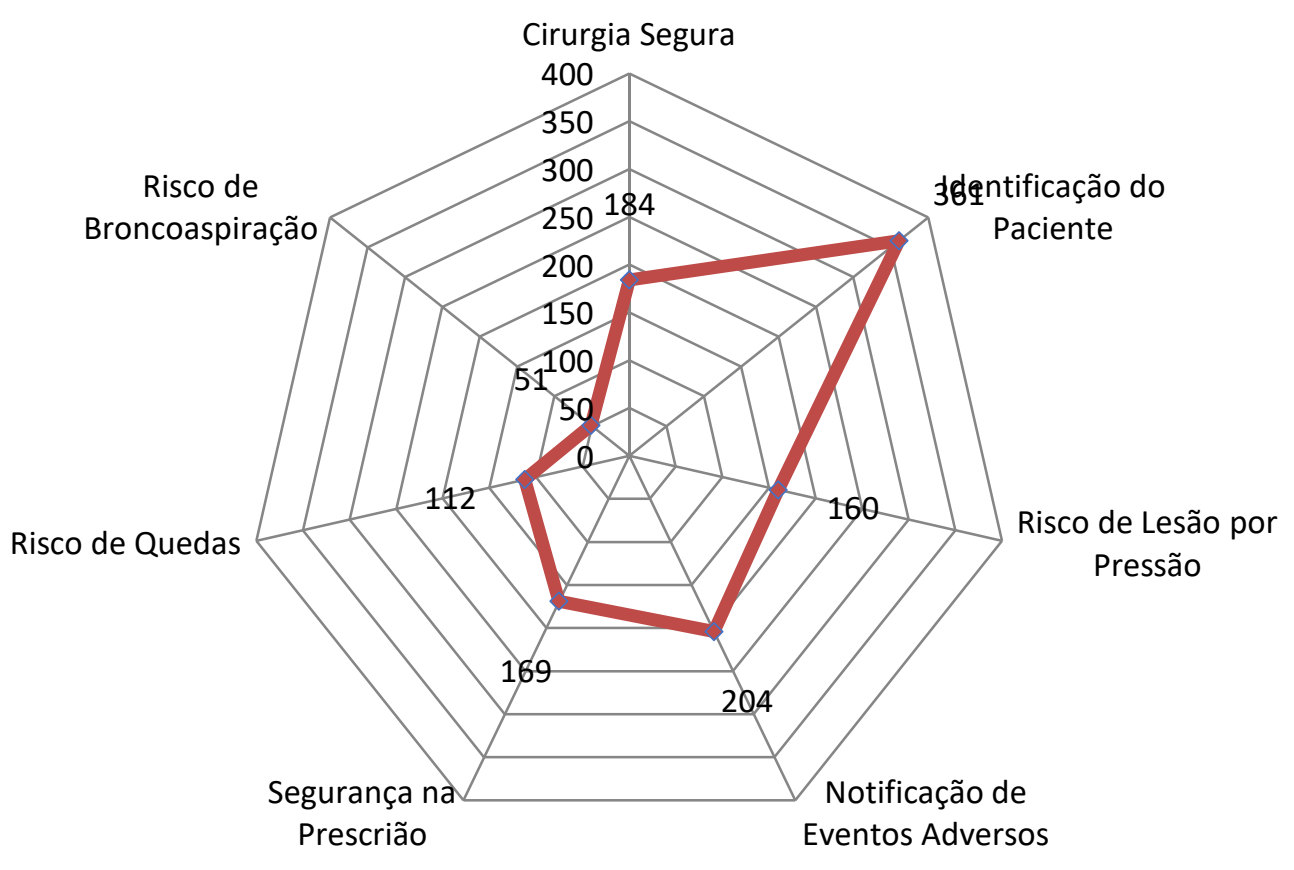

Fonte: Dados da pesquisa, 2017-2018.

Gráfico 2 - Quantidade de Classificação de Pacientes na Escala de Morse e Escala de Braden no Hospital Macrorregional Dra Ruth Noleto, 2018.

\section{CLASSIFICAÇÃO DO RISCO DE QUEDA E LESÃO POR PRESSÃO}

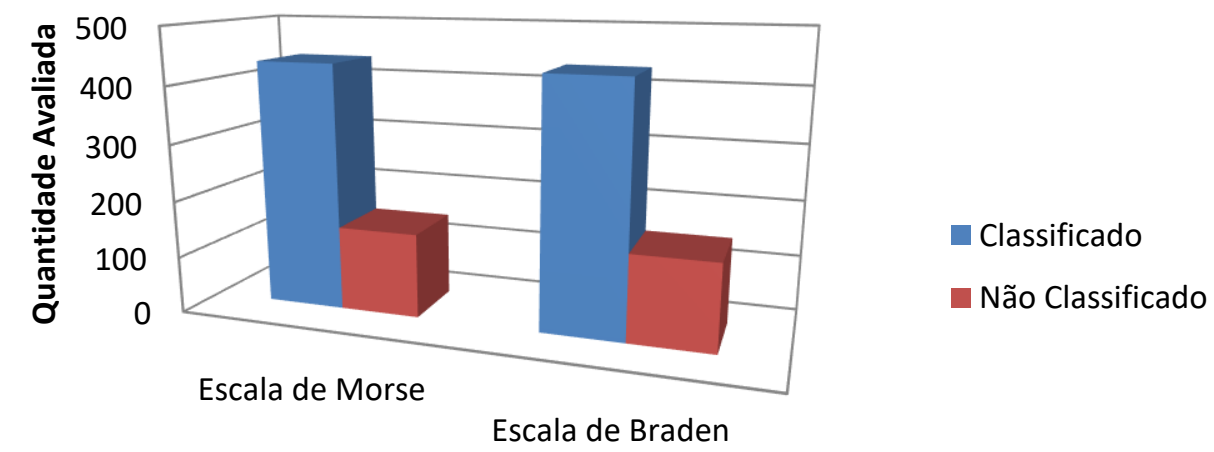

Escalas Avaliadas

Fonte: Dados da pesquisa, 2018. 
É válido pautar que o protocolo de Identificação do Paciente e Notificação de Eventos Adversos também foram fornecidos a outros profissionais da área hospitalar que não são da assistência direta ao paciente, são eles: administrativos, recepção, engenharia e limpeza.

O respeito a novas ideias e modos de trabalho, assim como a autonomia do profissional são fatores que contribuem para que o processo de ensino-aprendizagem seja eficaz, porém, a resistência a mudanças, excesso de serviço e o próprio trabalho são fatores de impedimento para este (PARANAGUÁ et al. 2016).

Como processo de avaliação da implantação de protocolo, foram criados alguns instrumentos para verificação diária dos setores, observando: aplicação da escala de Morse, aplicação da escala de Braden, uso de pulseiras vinculado à identificação de leito e uso do checklist de cirurgia segura.

Esta avaliação do risco de queda e lesão por pressão foi realizada mediante acompanhamento do sistema vigente (Gráfico 2). Todo paciente deveria ser classificado nestes dois instrumentos em até $24 \mathrm{~h}$ após a internação, depois do tempo transcorrido o responsável do NSP verificava tal sistema, a fim de saber se havia ou não classificação destes.

Assim, estes meios de implantação de controle são formas adaptadas para realidade desta instituição, podendo ser expandidas e redesenhadas de acordo com a tecnologia utilizada, necessidade dos serviços e até mesmo sem utilização de tecnologias de software.

\section{CONSIDERAÇÕES FINAIS}

Assim, depreende-se por intermédio deste relato de experiência, que o processo de instituição e implantação do Núcleo de Segurança do Paciente e seus protocolos é algo contínuo, variando de acordo com a realidade de cada instituição, bem como com as vivências próprias do processo ensino-aprendizagem. Necessitando de análise da participação de todos os atores envolvidos, desde a alta gestão do hospital, como, direções, coordenações, supervisores de setor e serviços, sendo necessária a participação ativa da linha de frente da assistência ao paciente. Em estudos posteriores podem ser observados a qualidade destes protocolos na prática diária dos profissionais de saúde, percebendo suas principais dificuldades de aplicação e os pontos mais comprometidos no desenvolvimento da garantia de uma assistência segura.

\section{REFERÊNCIAS}

1. ANVISA, Agência Nacional de Vigilância Sanitária. Boletim Segurança do Paciente e Qualidade em Serviços de Saúde $n^{\circ}$ 15: Incidentes Relacionados à Assistência à Saúde - 2016. Brasília; 2017.

2. AZEVEDO KCC et al. Implementation of the Patient Safety Core in a Health Service Implantación de Base de Seguridad del Paciente en un Servicio de Salud. Rev Enferm Ufpe On Line, Recife, v. 12, n. 10, p.4692-4695, dez. 2016.

3. BRASIL a, Ministério da Saúde (BR), Agência Nacional de Vigilância Sanitária. Resolução - RDC $N^{\circ} 36$, de 25 de Julho de 2013: Institui Ações para Segurança do Paciente em Serviços de Saúde e dá Outras Providências. Brasília (DF); 2013.

4. BRASIL b, Ministério da Saúde (BR), Gabinete do ministério. Portaria No 529 , de $1^{\circ}$ de Abril de 2013: Institui o Programa Nacional de Segurança do Paciente (PNSP). Brasília (DF); 2013.

5. BRASIL. Agência Nacional de Vigilância Sanitária. Implantação do Núcleo de Segurança do Paciente em Serviços de Saúde - Série Segurança do Paciente e Qualidade em Serviços de Saúde/Agência Nacional de Vigilância Sanitária Brasília: Anvisa, 2016.

6. CASSIANI SHB, et al. O Uso da Tecnologia para a Segurança do Paciente. Revista Eletrônica de Enfermagem, 2009; 11(2):413-7.

7. GAVA M et al. Incorporação da tecnologia da informação na Atenção Básica do SUS no Nordeste do Brasil: expectativas e experiências. Ciência \& Saúde Coletiva, 21(3):891-902, 2016.

8. OLIVEIRA RM et al. Estratégias para promover segurança do paciente: da identificação dos riscos às práticas baseadas em evidências. Escola Anna Nery: Revista de Enfermagem, 2014; 18(1) 122-129.

9. PARANAGUÁ TTB et al. Suporte para aprendizagem na perspectiva da segurança do paciente na atenção primária em saúde. Rev. Latino-Americana de Enfermagem, 2016; 24:e2771.

10. SIMAN AG, BRITO MJM. Mudanças na prática de enfermagem para melhorar a segurança do paciente. Revista Gaúcha de Enfermagem, [s.I.], v. 37, p.1-9, 2016. 